\title{
INCOMPRESSIBILITY AND NORMAL MINIMAL SURFACES
}

\author{
TEJAS KALELKAR
}

\begin{abstract}
In this paper we describe a procedure for refining the given triangulation of a 3-manifold that scales the PL-metric according to a given weight function while creating no new normal surfaces.

It is known that an incompressible surface $F$ in a triangulated 3-manifold $M$ is isotopic to a normal surface that is of minimal PL-area in the isotopy class of $F$. Using the above scaling refinement we prove the converse. If $F$ is a surface in a closed 3-manifold $M$ such that for any triangulation $\tau$ of $M, F$ is isotopic to a $\tau$-normal surface $F(\tau)$ that is of minimal PL-area in its isotopy class, then we show that $F$ is incompressible.
\end{abstract}

\section{INTRODUCTION}

Given a Riemannian manifold $(M, g)$, we can scale the metric by multiplying $g$ with a smooth positive real-valued function. Such a rescaling may, however, introduce new minimal surfaces. Given a triangulated 3 -manifold $(M, \tau)$, we can scale the PL-metric by taking a refinement of $\tau$, by repeatedly subdividing the tetrahedra in $\tau$ according to a positive integer-valued scaling function. In general, such a scaling may introduce new minimal normal surfaces. We describe here a procedure for scaling the PL-metric that introduces no new normal surfaces.

Definition 1.1. Let $\Delta$ be a tetrahedron with vertices labeled $\{a, b, c, d\}$. Let $e$ be a point in the interior of $\Delta$. Take a simplicial triangulation of $\Delta$ using the tetrahedra $\Delta_{A}=[b, c, d, e], \Delta_{B}=[a, c, d, e], \Delta_{C}=[a, b, d, e]$ and $\Delta_{D}=[a, b, c, e]$. Define $\phi$ on a triangulation $\tau$ to be the function that gives a refinement of $\tau$ by dividing each tetrahedron $\Delta$ of $\tau$ into 4 tetrahedra, as described above. We call this the refinement function. This is shown in Figure 1 where the additional edges in the refinement of $\Delta$ are shown as dotted-lines.

Let $f:\{\Delta: \Delta \in \tau\} \rightarrow \mathbb{Z}$ be a function that associates a non-negative integer to each tetrahedron $\Delta$ of $\tau$. We call such a function a scaling function. Define $\phi_{f}$ on $\tau$ to be the function that gives to each $\Delta \in \tau$ the triangulation $\phi^{f(\Delta)}(\Delta)$, obtained by taking $f(\Delta)$ iterates of $\phi$ on $\Delta$. As the faces of $\Delta$ are also faces of $\phi(\Delta), \phi_{f}(\tau)=\tau^{\prime}$ is a refined triangulation of $\tau$.

The main theorem in this paper is Theorem 1.2

Theorem 1.2. Let $F$ be a closed surface embedded in a 3-manifold $M$ no component of which is a 2-sphere. Let $\tau$ be a triangulation of $M$. Let $f:\{\Delta: \Delta \in \tau\} \rightarrow \mathbb{Z}$ be a scaling function and let $\tau^{\prime}=\phi_{f}(\tau)$ be the corresponding refinement of $\tau$. Then, $F$ is $\tau$-normal $\Leftrightarrow F$ is $\tau^{\prime}$-normal.

Date: March 2, 2022. 


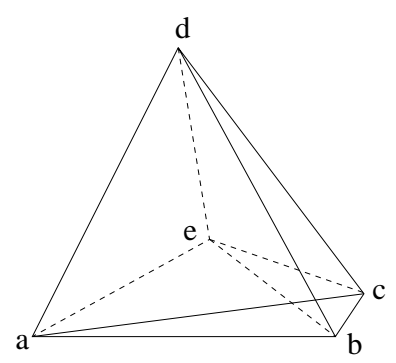

Figure 1. A tetrahedron in $\tau$, partitioned by $\phi$.

Every $\tau$-normal surface is $\tau^{\prime}$-normal by observing that each $\tau$-normal disk is a union of $\tau^{\prime}$-normal disks, as shown in lemma 2.5. For the converse, the proof depends on a simple examination of the possible $\tau^{\prime}$-normal proper embeddings of a surface in a tetrahedron $\Delta$ of the triangulation $\tau$. We show that every $\tau^{\prime}$-normal surface within $\Delta$ is in fact a $\tau$-normal disk, hence every $\tau^{\prime}$-normal surface is also $\tau$-normal.

In the second part of this paper we use such a refinement of the triangulation to obtain a PL-analogue of the theorem proved in [1. It is known that if $F$ is a smooth incompressible surface in an irreducible Riemannian 3-manifold $M$, then the isotopy class of $F$ has a least area surface. The theorem proved by Gadgil in [1] proves the converse, that is, if $F$ is a smooth surface in a closed, irreducible 3-manifold $M$ such that for each Riemannian metric $g$ of $M, F$ is isotopic to a least-area surface $F(g)$, then $F$ is incompressible.

Similarly, in the PL case, it is known that an incompressible surface $F$ in a triangulated 3-manifold $M$ is isotopic to a normal surface that is of minimal PLarea in the isotopy class of $F$. We prove here the converse.

Theorem 1.3. Let $F$ be a closed orientable surface in an irreducible orientable closed 3-manifold $M$. Then, $F$ is incompressible if and only if for any triangulation $\tau$ of $M$, there exists a $\tau$-normal surface $F(\tau)$ isotopic to $F$ that is of minimal $P L$ area in the isotopy class of $F$.

If $F$ is an incompressible surface that is not normal in a triangulation $\tau$ of $M$, then it is known that a PL-area decreasing isotopy exists. To prove the converse, we show that given a compressible surface $F$, there exists a triangulation $\tau^{\prime}$ for which the isotopy class of $F$ has no normal minimal surface. An outline of the proof is as follows. Let $\hat{F}$ be the surface obtained by compressing $F$ along a compressing disc. Therefore, $F$ is obtained from $\hat{F}$ by attaching a 1 -handle to $\hat{F}$. We start with a certain 'prism' triangulation of a regular neighbourhood $N(\hat{F})$ of $\hat{F}$ which is such that any connected normal surface lying in $N(\hat{F})$ is isotopic to a component of $\hat{F}$. We extend this triangulation to a triangulation $\tau$ of $M$. Let $\operatorname{Ar}(F)$ be the PL-Area of $F$ in $\tau$, then as the 1-handle can be chosen to avoid all edges of $\tau$, we always get a representative of $F$ (in its isotopy class) such that $\operatorname{Ar}(F)=\operatorname{Ar}(\hat{F})$.

We define a scaling function $f:\{\Delta: \Delta \in \tau\} \rightarrow \mathbb{Z}$ that takes the value 0 on $\Delta \subset N(\hat{F})$ and a value greater than $\operatorname{Ar}(\hat{F})$ for $\Delta$ not in $N(\hat{F})$. We now take the refinement $\tau^{\prime}$ of $\tau$ given by $\phi_{f}$. As every $\tau^{\prime}$-normal surface is also $\tau$-normal by Theorem 1.2 a $\tau^{\prime}$-normal surface that does not lie in $N(\hat{F})$ has a $\tau$-normal disk 
outside $N(\hat{F})$. This disk has $\tau^{\prime}$ PL-area more than the $\tau^{\prime}$ PL-area of $\hat{F}$. As $\hat{F}$ is not homeomorphic to $F$, a normal surface that lies entirely in $N(\hat{F})$ is not isotopic to $F$, while a normal surface that does not lie in $N(\hat{F})$ has $\tau^{\prime}$ PL-area more than that of $\hat{F}$. As there is always a surface isotopic to $F$ that has $\tau^{\prime}$ PL-area equal to that of $\hat{F}$ (and which is not normal) so, the isotopy class of $F$ has no normal minimal-area surfaces in $\tau^{\prime}$.

\section{Proof of Theorem 1.2}

In this section, we first show lemma 2.4 which determines normal disks using normal arcs in the boundary of the disk. Then, using Fig 2 we prove lemma 2.5 which says that a $\tau$-normal disk is a $\tau^{\prime}$-normal surface. We then prove Theorem 1.2. We introduce the following notation:

Definition 2.1. A $\tau^{\prime}$-normal triangle $T$ in $\Delta_{X} \subset \Delta, X \in\{A, B, C, D\}$, is said to link a vertex $w$ in $\Delta_{X}$ if $\partial \Delta_{X}-\partial T$ has a component that contains the vertex $w$ and no other vertices of $\Delta_{X}$. We say the coordinates of $T$ are $[T]=\left(X, T_{w}\right)$. When the context is clear we shall denote the triangle $T$ itself by its coordinates $\left(X, T_{w}\right)$.

Similarly, a $\tau^{\prime}$-normal quadrilateral $Q$ in $\Delta_{X} \subset \Delta, X \in\{A, B, C, D\}$, is said to link an edge $y z$ in $\Delta_{X}$ if $\partial \Delta_{X}-\partial Q$ has a component that contains the vertices $y$ and $z$, and no other vertices of $\Delta_{X}$. We say the coordinates of $Q$ are $[Q]=$ $\left(X, Q_{y z}\right)$. When the context is clear we shall denote the quadrilateral $Q$ itself by its coordinates $\left(X, Q_{y z}\right)$.

Definition 2.2. A $\tau^{\prime}$-normal arc $\lambda$ is said to link a vertex $x$ (respectively an edge $y z$ ) in a face $F$ of $\tau^{\prime}$ if $F-\lambda$ has a component that contains the vertex $x$ and no other vertices of $F$ (respectively contains the vertices $y$ and $z$ and no other vertices of $F$ ). Denote the set of $\tau^{\prime}$-normal arcs in faces of tetrahedra of $\tau^{\prime}$, linking vertex $x$ (respectively edge $y z$ ) by $\Lambda_{x}$ (respectively $\Lambda_{y z}$ ).

Definition 2.3. We define $\Lambda_{x} * \Lambda_{y}$ (respectively $\Lambda_{x} * \Lambda_{x y}$ ) to be the set of $\tau^{\prime}$-normal paths that are not contained in a single face, and are given by the concatenation of an $\operatorname{arc}$ in $\Lambda_{x}$ with an $\operatorname{arc}$ in $\Lambda_{y}$ (respectively $\Lambda_{x y}$ ).

We now state the following lemma which says that given a pair of contiguous normal arcs in the boundary of a normal disc, we can determine whether the disc is a triangle or a quadrilateral and we can determine which vertex (respectively which edge) it links. Also, if we are given that the normal disk is a triangle (respectively a quadrilateral) and we are given one normal arc in its boundary, then the vertex linked by the normal triangle (respectively the edge linked by the normal quadrilateral) can be determined.

Lemma 2.4. For a $\tau^{\prime}$-normal disk $D$ with a normal path $\lambda \subset \partial D$,

(i) If $D$ is a triangle with $\lambda \in \Lambda_{x}$ then $D$ is a triangle linking the vertex $x$.

(ii) If $D$ is a quadrilateral with $\lambda \in \Lambda_{x y}$ then $D$ is a quadrilateral linking the edge $x y$.

(iii) If $D$ is a quadrilateral in the tetrahedron $[w, x, y, z]$ with $\lambda$ in the face $[x, y, z]$ and $\lambda \in \Lambda_{z}$, then $D$ is a quadrilateral linking the edge wz.

(iv) If $\lambda \in \Lambda_{x} * \Lambda_{x}$ then $D$ is a triangle linking the vertex $x$.

(v) If $\lambda \in \Lambda_{x} * \Lambda_{x y}$ then $D$ is a quadrilateral linking the edge $x y$. 
Proof. We make the following simple observations:

a. A normal disk $D$ is a quadrilateral if and only if each normal arc in $\partial D$ links a distinct vertex, which is the same as saying normal arcs in $\partial D$ link more than one vertex.

b. A normal triangle $T$ in a tetrahedron $[w, x, y, z]$ links the vertex $x$ if and only if any normal arc in $\partial T$ links $x$.

c. A normal quadrilateral $Q$ in a tetrahedron $[w, x, y, z]$ links edge $x y$ if and only if $\partial Q \cap x y=\phi$.

The statement (i) follows from observation $\mathrm{b}$.

Let $D$ be a quadrilateral in a tetrahedron $[w, x, y, z]$, with $\lambda \in \Lambda_{x y}$ and $\lambda$ contained in the face $F=[x, y, z]$. Then $\partial \lambda$ is contained in the $\operatorname{arcs} x z$ and $y z$. Therefore, if $\partial D \cap x y \neq \phi$ then as $\partial D$ is transverse to edges, $\partial D$ is a circle in $\partial \Delta$ transversely intersecting each edge of the triangle $[x, y, z]$. Therefore, $\partial D$ must intersect some edge of this triangle more than once. This is a contradiction as $D$ is a normal disc, so that $\partial D$ intersects each edge at most once. Therefore, $\partial D \cap x y=\phi$ and statement (ii) follows from observation c.

Statement (iii) follows from a similar argument replacing $\Lambda_{x y}$ with $\Lambda_{z}$ and observing that quadrilaterals that link $x y$ are precisely the quadrilaterals that link $w z$.

Statement (iv) follows from observations a and b.

The disk $D$ in statement (v) is a quadrilateral from observation a. As there exists an arc $\lambda \subset \partial D$ with $\lambda \in \Lambda_{x y}$, from statement (ii) we can see that $D$ links the edge $x y$.

We now state the lemma which shows that every $\tau$-normal disk is a union of $\tau^{\prime}$-normal disks.

Lemma 2.5. Let $S$ be a properly embedded surface in $\Delta$. Then,

(i) $S$ is a $\tau^{\prime}$-normal surface with $S=\left(A, T_{d}\right) \cup\left(B, T_{d}\right) \cup\left(C, T_{d}\right)$ or $S=\left(A, Q_{d e}\right) \cup$ $\left(B, Q_{d e}\right) \cup\left(C, Q_{d e}\right) \cup\left(D, T_{e}\right) \Leftrightarrow S$ is $\tau$-isotopic to a $\tau$-normal triangle linking vertex $d$.

(ii) $S$ is a $\tau^{\prime}$-normal surface with $S=\left(D, T_{a}\right) \cup\left(B, Q_{a d}\right) \cup\left(C, Q_{a d}\right) \cup\left(A, T_{d}\right)$ or $S=\left(B, T_{c}\right) \cup\left(D, Q_{b c}\right) \cup\left(A, Q_{b c}\right) \cup\left(C, T_{b}\right) \Leftrightarrow S$ is $\tau$-isotopic to a $\tau$-normal quadrilateral linking edge ad.

(iii) $S$ is a $\tau^{\prime}$-normal surface with $S=\left(A, T_{e}\right) \cup\left(B, T_{e}\right) \cup\left(C, T_{e}\right) \cup\left(D, T_{e}\right) \Leftrightarrow S$ is $a \tau^{\prime}$ vertex-linking sphere linking the vertex $e$.

Proof. If $S$ is a $\tau$-normal triangle in the tetrahedron $\Delta$, linking vertex $d$, then after a $\tau$-normal isotopy we may assume that $S=\partial B(d) \cap \Delta$, where $B(d)$ is a small ball neighbourhood of $d$ in $M$. This is shown in Figure 2 (i) a. Then, $S$ intersects the faces of $\tau^{\prime}$ as shown in Figure 2 (i) b, so that $S=\left(A, T_{d}\right) \cup\left(B, T_{d}\right) \cup\left(C, T_{d}\right)$. Conversely, if $S=\left(A, T_{d}\right) \cup\left(B, T_{d}\right) \cup\left(C, T_{d}\right)$ (Figure 2 (i) b) or $S=\left(A, Q_{d e}\right) \cup$ $\left(B, Q_{d e}\right) \cup\left(C, Q_{d e}\right) \cup\left(D, T_{e}\right)$ (Figure 2 (ii) b.), then $S$ is a properly embedded disk in $\Delta$ with $\partial S$ a circle in $\partial \Delta$ linking vertex $d$. So, $S$ is a $\tau$-normal triangle linking vertex $d$.

If $S$ is a $\tau$-normal quadrilateral in the tetrahedron $\Delta$, linking edge $a d$, then after a $\tau$-normal isotopy we may assume that $S=\partial B(a d) \cap \Delta$, where $B(a d)$ is a small ball neighbourhood of $a d$ in $M$. This is shown in Figure2(iii) a. Then, $S$ intersects 

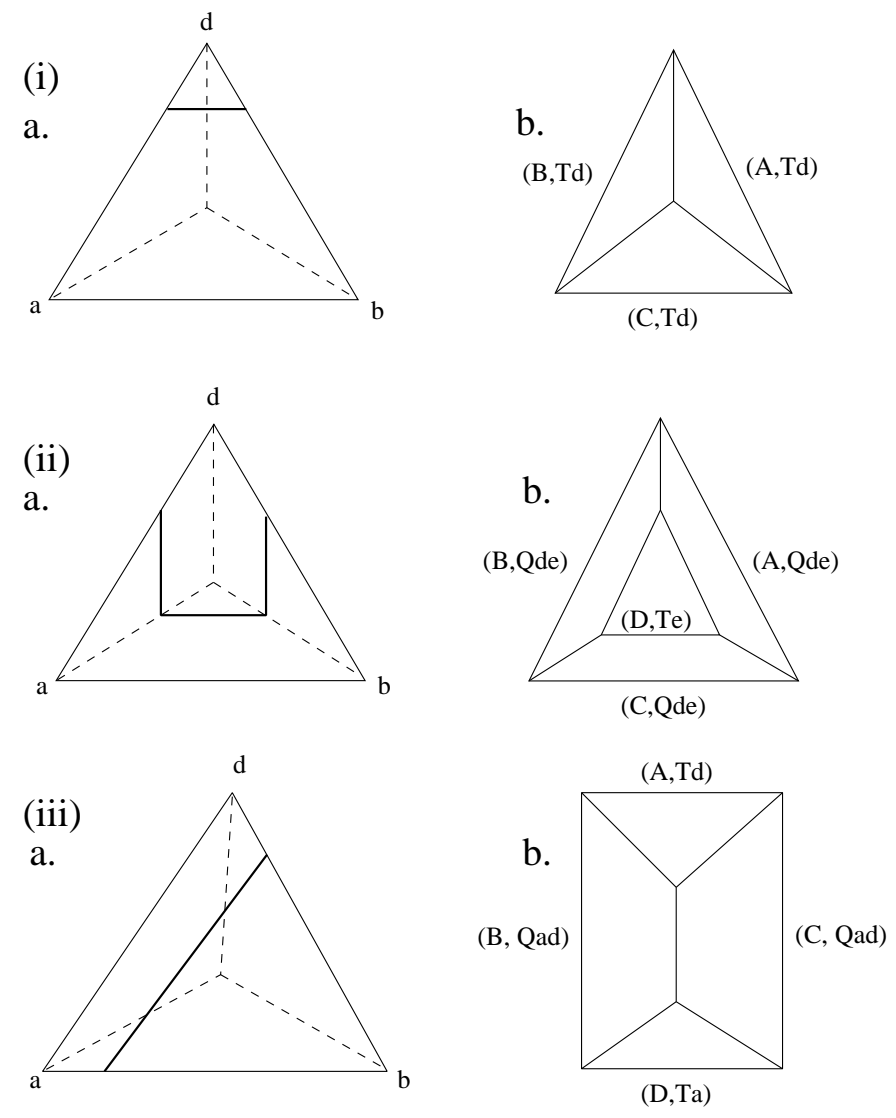

Figure 2. Diagrams (i) and (ii) represent a normal triangle linking vertex d. Diagram (iii) represents a normal quadrilateral linking edge ad.

the faces of $\tau^{\prime}$ as shown in Figure2(iii) b, so that $S=\left(D, T_{a}\right) \cup\left(B, Q_{a d}\right) \cup\left(C, Q_{a d}\right) \cup$ $\left(A, T_{d}\right)$. Conversely if $S=\left(D, T_{a}\right) \cup\left(B, Q_{a d}\right) \cup\left(C, Q_{a d}\right) \cup\left(A, T_{d}\right)$ (Figure 2 (iii) b) or $S=\left(B, T_{c}\right) \cup\left(D, Q_{b c}\right) \cup\left(A, Q_{b c}\right) \cup\left(C, T_{b}\right)$ (corresponding to a quadrilateral linking the edge $b c$ ), then $S$ is a properly embedded disk in $\Delta$ with $\partial S$ a circle in $\partial \Delta$ linking the edge $a d$. So, $S$ is a $\tau$-normal quadrilateral linking edge $a d$.

If $S$ is a vertex linking sphere linking vertex $e$, then $S=\partial B(e)$ where $B(e)$ is a small ball-neighbourhood of $e$ in $M$. So that $S=\left(A, T_{e}\right) \cup\left(B, T_{e}\right) \cup\left(C, T_{e}\right) \cup\left(D, T_{e}\right)$. Conversely, if $S=\left(A, T_{e}\right) \cup\left(B, T_{e}\right) \cup\left(C, T_{e}\right) \cup\left(D, T_{e}\right)$ then it is easy to see that $S=\partial B(e)$ and therefore $S$ is a vertex-linking sphere in $\tau^{\prime}$ linking vertex $e$.

We now give a proof of Theorem 1.2 ,

Proof. If $F$ is $\tau$-normal then by lemma 2.5 it follows that $F$ is a union of $\tau^{\prime}$-normal disks and hence is $\tau^{\prime}$-normal as well.

To prove the converse, let $S$ be a connected component of $F \cap \Delta$. We shall show in Claims 1 and 2 that if $S$ contains a $\tau^{\prime}$-normal triangle, then $S$ must either be a 
$\tau$-normal disk or a vertex-linking sphere. In Claim 3 we shall show that $S$ is not the union of $\tau^{\prime}$-normal quadrilaterals. So every component of $F \cap \Delta$ is either a $\tau$-normal disk or a $\tau^{\prime}$ vertex-linking sphere. Thus we would have shown that any $\tau^{\prime}$-normal surface in $M$ is either a $\tau$-normal surface or it has a component which is a $\tau^{\prime}$ vertex-linking sphere.

Claim 1. If $S^{\prime} \subset S$ is a $\tau^{\prime}$-normal triangle with coordinates $\left(X, T_{e}\right)$ for some $X \in\{A, B, C, D\}$ then $S$ is either a vertex linking sphere in $\tau^{\prime}$ linking vertex $e$ or $S$ is a $\tau$-normal triangle.

Without loss of generality, assume $X=D$. The boundary $\partial S^{\prime}$ is composed of normal arcs linking vertex $e$, i.e., for $Y \in\{A, B, C\}, S^{\prime} \cap \Delta_{Y}$ gives a normal arc in $\Lambda_{e}$.

If $S^{\prime} \cap \Delta_{A}$ meets a $\tau^{\prime}$-normal triangle $T$ in $\Delta_{A}$, then by lemma 2.4 (i), [T]= $\left(A, T_{e}\right)$. Let $S^{\prime \prime}=S^{\prime} \cup T$. Now for $Y \in\{B, C\} ; S^{\prime \prime} \cap \Delta_{Y} \in \Lambda_{e} * \Lambda_{e}$, therefore by lemma 2.4 (iv) $S^{\prime \prime}$ meets normal triangles with coordinates $\left(B, T_{e}\right)$ and $\left(C, T_{e}\right)$. So as $S$ is connected, $S=\left(A, T_{e}\right) \cup\left(B, T_{e}\right) \cup\left(C, T_{e}\right) \cup\left(D, T_{e}\right)$, therefore by lemma 2.5. $S$ is a vertex-linking sphere linking vertex $e$.

If $S^{\prime} \cap \Delta_{A}$ meets a $\tau^{\prime}$-normal quadrilateral $Q$ in $\Delta_{A}$, then by lemma 2.4 (iii), $[Q]=\left(A, Q_{d e}\right)$. Let $S^{\prime \prime}=S^{\prime} \cup Q$. Now for $Y \in\{B, C\} ; S^{\prime \prime} \cap \Delta_{Y} \in \Lambda_{e} * \Lambda_{d e}$, therefore by lemma $2.4(\mathrm{v}), S^{\prime \prime}$ meets normal quadrilaterals with coordinates $\left(B, Q_{d e}\right)$ and $\left(C, Q_{d e}\right)$. So as $S$ is connected, $S=\left(A, Q_{d e}\right) \cup\left(B, Q_{d e}\right) \cup\left(C, Q_{d e}\right) \cup\left(D, T_{e}\right)$, therefore by lemma 2.5. $S$ is a $\tau$-normal triangle (linking vertex $d$ ).

Claim 2. If $S^{\prime} \subset S$ is a $\tau^{\prime}$-normal triangle with coordinates $\left(X, T_{w}\right)$, for $X \in$ $\{A, B, C, D\}$, where $w$ is a vertex in $\Delta_{X}$ other than $e$, then $S$ is either a $\tau$-normal triangle or a $\tau$-normal quadrilateral.

Without loss of generality, assume $X=D$ and $w=a$. Then $S^{\prime} \cap \Delta_{B}$ and $S^{\prime} \cap \Delta_{C}$ are in $\Lambda_{a}$, while $S^{\prime} \cap \Delta_{A}=\phi$.

If $S^{\prime} \cap \Delta_{B}$ meets a $\tau^{\prime}$-normal triangle $T$, then by lemma[2.4(i), [T] $=\left(B, T_{a}\right)$. Let $S^{\prime \prime}=S^{\prime} \cup T$. Then $S^{\prime \prime} \cap \Delta_{C} \in \Lambda_{a} * \Lambda_{a}$ therefore by lemma2.4(iv), $S^{\prime \prime}$ meets a normal triangle with coordinates $\left(C, T_{a}\right)$ in $\Delta_{C}$. So we have $S=\left(D, T_{a}\right) \cup\left(B, T_{a}\right) \cup\left(C, T_{a}\right)$, therefore by Lemma 2.5, $S$ is a $\tau$-normal triangle (linking vertex $a$ ).

If $S^{\prime} \cap \Delta_{B}$ meets a $\tau^{\prime}$-normal quadrilateral $Q$, then by lemma 2.4 (iii), $[Q]=$ $\left(B, Q_{a d}\right)$. We have $\left(S^{\prime} \cup Q\right) \cap \Delta_{C} \in \Lambda_{a} * \Lambda_{a d}$ therefore by lemma 2.4 (v), $S^{\prime} \cup Q$ meets $\Delta_{C}$ in a quadrilateral $Q^{\prime}$ with $\left[Q^{\prime}\right]=\left(C, Q_{a d}\right)$. Let $S^{\prime \prime}=S \cup Q \cup Q^{\prime}$. Then $S^{\prime \prime} \cap \Delta_{A} \in \Lambda_{d} * \Lambda_{d}$. So that by lemma 2.4 (iv), $S^{\prime \prime}$ meets $\Delta_{A}$ in a normal triangle with coordinates $\left(A, T_{d}\right)$. Therefore $S=\left(D, T_{a}\right) \cup\left(B, Q_{a d}\right) \cup\left(C, Q_{a d}\right) \cup\left(A, T_{d}\right)$ and by Lemma 2.5. $S$ is a $\tau$-normal quadrilateral (linking edge $a d$ ).

Claim 3. $S$ is not a union of $\tau^{\prime}$-normal quadrilaterals.

Without loss of generality we assume $S \cap \Delta_{D} \neq \phi$. Let $Q$ be a normal quadrilateral in $S \cap \Delta_{D}$. Then as the normal arcs in $\partial Q$ link distinct vertices, there exists an $\operatorname{arc} \lambda \subset \partial Q$ that belongs to $\Lambda_{e}$. Assume, without loss of generality, that $Q \cap \Delta_{A}=\lambda \in \Lambda_{e}$. By lemma 2.4 (iii), $Q$ meets $\Delta_{A}$ in a normal quadrilateral $Q_{A}$ with $\left[Q_{A}\right]=\left(A, Q_{d e}\right)$. As $Q_{A} \cap \Delta_{B} \in \Lambda_{d e}$, by lemma 2.4 (ii), $Q_{A}$ meets $\Delta_{B}$ in quadrilateral $Q_{B}$ with $Q_{B}=\left(B, Q_{d e}\right)$. Let $S^{\prime}=Q_{A} \cup Q_{B}$. Then $S^{\prime} \cap \Delta_{D} \in \Lambda_{e} * \Lambda_{e}$, 
so by lemma 2.4 (iv), $S^{\prime}$ must meet $\Delta_{D}$ in a triangle with coordinates $\left(D, T_{e}\right)$ contradicting our assumption that $S$ is composed solely of $\tau^{\prime}$-normal quadrilaterals.

\section{The PRism triangulation of $\mathrm{N}(\mathrm{F})$}

Let $F$ be a closed oriented connected surface lying in an oriented 3-manifold $M$. Denote by $I$ the closed interval $[-1,1]$. Let $N(F) \cong F \times I$ be a regular neighbourhood of $F$. In this section we define a triangulation of $N(F)$, which is such that any closed connected normal surface lying in $N(F)$ is normally isotopic to $F \times\{0\}$.

Take a triangular disc $T$ with oriented edges. Assume the edges are not cyclically oriented. Label the vertices $\left\{v_{0}, v_{1}, v_{2}\right\}$ of $T$ in such a way that the edges are oriented as $\left\{v_{0} v_{1}, v_{1} v_{2}, v_{0} v_{2}\right\}$. In $T \times I$, let $T \times\{-1\}$ be identified with $\left[v_{0}, v_{1}, v_{2}\right]$ labeled as above and $T \times\{1\}=\left[w_{0}, w_{1}, w_{2}\right]$, where $v_{i}$ and $w_{i}$ have the same image under the projection $T \times I \rightarrow T$. Then we get a triangulation of $T \times I$, using the tetrahedra $\Delta_{0}=\left[v_{0}, w_{0}, w_{1}, w_{2}\right], \Delta_{1}=\left[v_{0}, v_{1}, w_{1}, w_{2}\right]$ and $\Delta_{2}=\left[v_{0}, v_{1}, v_{2}, w_{2}\right]$. We call this the prism triangulation of $T \times I$. (See proof of Theorem 2.10 [2] for details.)

Lemma 3.1. Let $T_{1}$ and $T_{2}$ be triangles with non-cyclic oriented edges that intersect in an edge $e=T_{1} \cap T_{2}$. Assume the orientation on the edge e coming from $T_{1}$ is the same as that coming from $T_{2}$. Let $\tau_{1}$ and $\tau_{2}$ be the prism triangulations of $T_{1} \times I$ and $T_{2} \times I$ respectively. Then $\tau=\tau_{1} \cup \tau_{2}$ is a triangulation of $\left(T_{1} \cup T_{2}\right) \times I$.

Proof. In the prism triangulation of $\mathrm{T}$, we note that the 1-skeleton lies in $\partial T \times I$ and is the union of $\partial T \times \partial I$ and the edges $\left\{v_{0} w_{0}, v_{1} w_{1}, v_{2} w_{2}, v_{0} w_{1}, v_{0} w_{2}, v_{1} w_{2}\right\}$. Recall that the edges of $T$ were oriented as $\left\{v_{0} v_{1}, v_{1} v_{2}, v_{0} v_{2}\right\}$. So given an oriented edge $e=[-1,1]$ of $T$, with $e$ oriented in the direction from -1 to $1, e \times I$ is the union of two triangles given by the join of $e \times\{-1\}$ with the point $(1,1)$ and the join of $e \times\{1\}$ with the point $(-1,-1)$. In particular, the triangles divide the square $e \times I$ along the diagonal from $(-1,-1)$ to $(1,1)$.

Therefore if two triangles $T_{1}$ and $T_{2}$ with oriented edges intersect in an edge $e=T_{1} \cap T_{2}$, where the orientation of $e$ coming from $T_{1}$ is the same as that from $T_{2}$, then the prism triangulation of $T_{1} \times I$ and $T_{2} \times I$ agree on the intersection $e \times I$. So by taking the union $\tau_{1} \cup \tau_{2}$ we get a triangulation on $\left(T_{1} \cup T_{2}\right) \times I$.

We can now define the prism triangulation on $F \times I$. Firstly, we claim that given a triangulation $\tau$ of $F$ there exists a refinement $\tau^{\prime}$ of $\tau$ and an orientation of the edges of $\tau^{\prime}$ such that no triangle has edges oriented cyclically.

Give any orientation to the edges of the 1-skeleton of $\tau$. Let $N_{\tau}$ be the number of triangles of $\tau$ with edges oriented cyclically. If $N_{\tau}>0$, then take a triangle $T=[a, b, c]$ in $\tau$ with cyclically oriented edges $\{a b, b c, c a\}$. Let $d$ be a point in the interior of $T$. Define the triangulation $\tau^{\prime}$ as a refinement of $\tau$ given by subdividing $T$ into the triangles $[a, b, d],[b, c, d],[c, a, d]$. Orient the newly introduced edges of the 1-skeleton as $d a, d b$ and $d c$. Then none of the triangles in the subdivision of $T$ has cyclically oriented edges. Therefore the number of triangles with cyclically oriented edges in $\tau^{\prime}, N_{\tau^{\prime}}=N_{\tau}-1$. So after $N_{\tau}$ such refinements we obtain a triangulation of $F$ with no triangles having edges oriented cyclically.

Now by lemma 3.1. we can patch up the prism triangulations of triangles of $F$ to get a triangulation of $F \times I$. We call this the prism triangulation of $F \times I$, relative 
to the triangulation $\tau^{\prime}$ of $F$.

We now show that any properly embedded normal surface in the prism triangulation of $T \times I$ with boundary in $\partial T \times I$ is normally isotopic to $T \times\{0\}$.

Lemma 3.2. Let $T=\left[v_{0}, v_{1}, v_{2}\right]$ be a triangle with non-cyclic edges. Let $\tau$ be the prism triangulation on $T \times I$. Let $S$ be a properly embedded normal surface with $\partial S \subset \partial T \times I$. Then $S$ is normally isotopic to $T \times\{0\}$.

Proof. In the prism $T \times I$, let $T \times\{-1\}=\left[v_{0}, v_{1}, v_{2}\right]$ and $T \times\{1\}=\left[w_{0}, w_{1}, w_{2}\right]$ with $v_{i}$ and $w_{i}$ projecting to the same point on $T$. Then, the prism triangulation of $T \times I$ is composed of the tetrahedra $\Delta_{0}=\left[v_{0}, w_{0}, w_{1}, w_{2}\right], \Delta_{1}=\left[v_{0}, v_{1}, w_{1}, w_{2}\right]$ and $\Delta_{2}=\left[v_{0}, v_{1}, v_{2}, w_{2}\right]$.

Observe that $\Delta_{0}$ contains the face $\left[w_{0} w_{1} w_{2}\right]=T \times\{1\}$, while $\Delta_{2}$ contains the face $\left[v_{0} v_{1} v_{2}\right]=T \times\{-1\}$. As $S$ does not intersect $T \times \partial I, S \cap \Delta_{0}$ is parallel to $\left[w_{0} w_{1} w_{2}\right]$ and is therefore a union of triangles linking $v_{0}$. Similarly, $S \cap \Delta_{2}$ is a union of triangles linking $w_{2}$. The tetrahedron $\Delta_{1}$ has a pair of opposing edges $v_{0} v_{1}$ and $w_{1} w_{2}$ that lie in $T \times \partial I$. Therefore $S \cap \Delta_{1}$ is a union of normal disks that separates these pair of edges and is therefore a union of normal quadrilaterals linking edge $v_{0} v_{1}$.

Note that $\Delta_{0} \cap \Delta_{1}=\left[v_{0} w_{1} w_{2}\right]$ and $\Delta_{1} \cap \Delta_{2}=\left[v_{0} v_{1} w_{2}\right]$. So by the matching equations, the number of triangles in $\Delta_{0} \cap S$ equals the number of quadrilaterals in $\Delta_{1} \cap S$ which is the same as the number of triangles in $\Delta_{2} \cap S$.

Let $T_{0}$ be a triangle in $\Delta_{0} \cap S, Q_{1}$ a quadrilateral in $\Delta_{1} \cap S$ and $T_{2}$ a triangle in $\Delta_{2} \cap S$ such that $T_{0}$ meets $Q_{1}$ in $\Delta_{0} \cap \Delta_{1}$ and $Q_{1}$ meets $T_{2}$ in $\Delta_{1} \cap \Delta_{2}$. Then $T_{0} \cup Q_{1} \cup T_{2}=S^{\prime}$ is a connected properly embedded normal surface in $T \times I$ that projects homeomorphically onto $T$ and so $S^{\prime}$ is normally isotopic to $T \times\{0\}$. Any normal surface in $T \times I$ that does not intersect $T \times \partial I$ is therefore, a disjoint union of discs parallel to $T \times\{0\}$. As $S$ is a normal connected surface, $S=S^{\prime}$ as required.

Theorem 3.3. Let $F$ be a closed oriented connected surface. Let $\tau$ be a prism triangulation of $N(F) \simeq F \times I$. Then any normal closed connected surface $F^{\prime} \subset$ $N(F)$ is normally isotopic to $F \times\{0\}$.

Proof. Let $\tau^{\prime}$ be a triangulation of $F$, and let $\tau$ be the prism triangulation of $N(F)$ relative to $\tau^{\prime}$. Let $T$ be a triangle in $\tau^{\prime}$ then $\left.\tau\right|_{T}$ is the prism triangulation on $T \times I$. As $F^{\prime}$ is normal in $\tau$ and is closed, $F^{\prime} \cap(T \times I)$ is a $\left.\tau\right|_{T^{-} \text {-normal properly embedded }}$ surface $S$ with $\partial S \subset \partial T \times I$ so by lemma 3.2, $S$ is normally isotopic to $T \times\{0\}$. As this is true for every triangle $T$ in the triangulation $\tau^{\prime}$ of $F$ and the surface $F^{\prime}$ is connected, $F^{\prime}$ is normally isotopic to $F \times\{0\}$.

\section{Proof of Theorem 1.3}

As before, let $F$ be a closed oriented surface in a compact oriented 3-manifold $M$. Let $\tau_{1}$ be a triangulation of $N(F)$. In this section we firstly show that given any integer $W$, there exists an extension of $\tau_{1}$ to a triangulation $\tau$ of $M$ such that any $\tau$-normal surface $F^{\prime}$ that does not lie in $N(F)$ has PL-area more than $W$. This is shown in lemma 4.4 , using which we prove Theorem 1.3 . 
Definition 4.1. Let $\Gamma$ be a simplicial complex of dimension $n$. Then $|\Gamma|$ denotes the number of $n$-cells in $\Gamma$.

Definition 4.2. Let $\tau$ be a triangulation of $M$. Let the $i$-weight of $F$ be defined as $w^{(i)}(F)=\left|F \cap \tau^{(i)}\right|$, where $\tau^{(i)}$ is the $i$-the skeleton of the triangulation $\tau$. Then, the PL-area of $F$ is given by the ordered pair $w(F)=\left(w^{(1)}(F), w^{(2)}(F)\right)$.

Lemma 4.3. Let $\phi$ be the refinement function (Definition 1.1) that gives the refinement of a tetrahedron $\Delta$ into 4 tetrahedra. Let $\tau$ be a triangulation of $\Delta$ consisting of the single tetrahedron $\Delta$. Let $\tau^{n}=\phi^{n}(\tau)$ be a triangulation of $\Delta$ obtained by taking $n$ iterates of $\phi$. Let $D$ be a $\tau$-normal disk. Then the 1-weight of $D$ in $\tau^{n}$ is greater than $n$.

Proof. As $D$ is $\tau$-normal, by Theorem 1.2, $D$ is $\tau^{n}$ - normal. Let $d_{n}$ be the number of $\tau^{n}$-normal disks in $D$. Let $w_{n}(D)=w^{(1)}(D)$ in $\tau^{n}$, be the 1 -weight of $D$ in $\tau^{n}$. As $D$ is a $\tau$-normal disk, its weight in $\tau^{0}=\tau$ is greater than equal to 3 , therefore $d_{0}=1$ and $w_{0}(D) \geq 3$. Now we claim that for $n>0, d_{n} \geq 3 d_{n-1}$ and $w_{n}(D) \geq w_{n-1}(D)+d_{n-1}$.

By lemma 2.5. $D$ is divided into at least $3 \tau^{1}$-normal disks on taking the refinement along $\phi$, and its weight is increased by at least one. Therefore $d_{1} \geq 3 d_{0}$ and $w_{1} \geq w_{0}+d_{0}$.

Similarly now, if $D$ is the union of $d_{n-1} \tau^{n-1}$-normal disks then each such disk is divided into at least $3 \tau^{n}$-normal disks by taking the refinement along $\phi$, while its weight is incremented by at least one for each of the $\tau^{n-1}$-normal disks. Therefore $d_{n} \geq 3 d_{n-1}$, while $w_{n} \geq w_{n-1}+d_{n-1}$. So by induction, $w_{n} \geq w_{0}+\sum_{i=0}^{n-1} d_{i} \geq$ $w_{0}+\left(\sum_{i=0}^{n-1} 3^{i}\right) d_{0}$

Therefore the weight $w_{n}(D) \geq 3+1+3+3^{2}+3^{3} \ldots+3^{n-1}>n$ for all $n>0$.

Lemma 4.4. Let $\hat{F}$ be a closed surface in $M$ and let $W$ be a positive integer. Let $\tau_{1}$ be a triangulation of a regular neighbourhood $N(\hat{F})$ of $\hat{F}$ in $M$. Then, there exists an extension of $\tau_{1}$ to a triangulation $\tau$ of $M$ such that for any $\tau$-normal surface $S$ that is not contained in $N(\hat{F}), w^{(1)}(S)>W$.

Proof. We extend the triangulation of $\partial N(\hat{F})$ given by $\tau_{1}$, to a triangulation $\tau_{2}$ of $M-\operatorname{int}(N(\hat{F}))$. Then, $\tau^{\prime}=\tau_{1} \cup \tau_{2}$ is a triangulation of $M$. Let $f$ be the scaling function that takes the value $W$ on tetrahedra of $\tau_{2}$ and the value 0 on tetrahedra of $\tau_{1}$. Let $\tau=\phi_{f}\left(\tau^{\prime}\right)$ be the corresponding refined triangulation. We claim that $\tau$ is the required triangulation.

Let $S$ be a $\tau$-normal surface in $M$ that is not contained in $N(S)$. By Theorem 1.2 then, $S$ is $\tau^{\prime}$-normal as well. As $S$ is not contained in $N(\hat{F})$ there exists a $\tau_{2}$-normal disk $D$ in $S-\operatorname{int}(N(\hat{F}))$. By lemma 4.3 now, the 1 -weight of $D$ in $\tau$ is greater than $W$, therefore $w^{(1)}(S)>W$ in $\tau$.

We are now in a position to prove Theorem 1.3 .

Proof. Assume $F$ is incompressible. Let $\tau$ be any triangulation of $M$. Let $F^{\prime}$ be a surface isotopic to $F$ of minimal PL-area in the isotopy class of $F$. If $F^{\prime}$ is not $\tau$-normal then it is known that there exists a weight minimising isotopy of $F^{\prime}$, which is a contradiction. So every minimal PL-area surface in the isotopy class of $F$ is normal. 
Conversely, suppose $F$ is compressible. Let $\hat{F}$ be the surface obtained by compressing $F$ along a compressing disk. The surface $F$ is obtained from $\hat{F}$ by attaching a 1-handle $\gamma$.

Let $\tau^{\prime}$ be a prism triangulation of $N(\hat{F}) \cong \hat{F} \times I$. Let the 1-weight of the normal surface $\hat{F} \times\{0\}$ be denoted by $W$. By applying lemma 4.4, we obtain an extension of $\tau^{\prime}$ to a triangulation $\tau$ of $M$ such that any normal surface that does not lie in $N(\hat{F})$ has 1-weight greater than $W$.

We can assume the 1-handle $\gamma$ is disjoint from the 1-skeleton of $\tau$. As $F$ is obtained from $\hat{F} \times\{0\}$ by attaching this 1-handle, the 1 -weight $W=w^{(1)}(\hat{F} \times\{0\})=$ $w^{(1)}(F)$.

Assume there exists a normal minimal surface $F^{\prime}$ isotopic to $F$. By construction of $\tau$, any normal surface that does not lie in $N(\hat{F})$ has 1-weight more than $W=$ $w(F)$. So, $F^{\prime}$ lies in $N(\hat{F})$.

By Theorem 3.3 then, $F^{\prime}$ is isotopic to a connected component of $\hat{F}$. As $F^{\prime}$ is isotopic to $F$, we have $F$ isotopic to a connected component of $\hat{F}$. This is a contradiction as $F$ is compressible and hence every component of $\hat{F}$ has genus strictly lower than the genus of $F$.

Acknowledgements. The author would like to thank Siddhartha Gadgil for useful discussions and advice. The CSIR-SPM Fellowship is acknowledged for financial support.

\section{REFERENCES}

1. Gadgil, Incompressibility and least-area surfaces, Expo. Math. 26 (2008), no. 1, 93-98. 57N10 (53Axx)

2. Hatcher, Algebraic Topology, Cambridge University Press, 2002

Stat-Math Unit, Indian Statistical Institute, Bangalore, India

E-mail address: tejas@isibang.ac.in 